SPbU IP-98-4, 13 January 98, chao-dyn/9801033, 27 January 98

\title{
Renormalization Group, Operator Product Expansion, and Anomalous Scaling in a Model of Advected Passive Scalar
}

\author{
Loran Ts. Adzhemyan \\ Nikolaj V. Antonov \\ Alexander N. Vasil'ev \\ Department of Theoretical Physics, St Petersburg University, \\ Uljanovskaja 1, St Petersburg-Petrodvorez, 198904, Russia
}

\begin{abstract}
Field theoretical renormalization group methods are applied to the ObukhovKraichnan model of a passive scalar advected by the Gaussian velocity field with the covariance

$$
\left\langle\mathbf{v}(t, \mathbf{x}) \mathbf{v}\left(t^{\prime}, \mathbf{x}\right)\right\rangle-\left\langle\mathbf{v}(t, \mathbf{x}) \mathbf{v}\left(t^{\prime}, \mathbf{x}^{\prime}\right)\right\rangle \propto \delta\left(t-t^{\prime}\right)\left|\mathbf{x}-\mathbf{x}^{\prime}\right|^{\varepsilon}
$$

Inertial range anomalous scaling for the structure functions and various pair correlators is established as a consequence of the existence in the corresponding operator product expansions of "dangerous" composite operators [powers of the local dissipation rate], whose negative critical dimensions determine anomalous exponents. The main technical result is the calculation of the anomalous exponents in the order $\varepsilon^{2}$ of the $\varepsilon$ expansion. Generalization of the results obtained to the case of a "slow" velocity field is also presented.
\end{abstract}

PACS number(s): 47.10.+g, 47.25.Cg, 05.40.+j 


\section{INTRODUCTION}

One of the main problems in the modern theory of fully developed turbulence is to verify the basic principles of the Kolmogorov-Obukhov (KO) phenomenological theory [1,2] within the framework of a microscopic model and to investigate deviations from this theory, provided they exist.

In particular, one is interested in the single-time "structure functions"

$$
S_{n}(r) \equiv\left\langle\left[\theta(\mathbf{x})-\theta\left(\mathbf{x}^{\prime}\right)\right]^{n}\right\rangle, \quad r \equiv\left|\mathbf{x}-\mathbf{x}^{\prime}\right|
$$

in the inertial range. Here $\theta(x) \equiv \theta(t, \mathbf{x})$ can be the component of the velocity field directed along the vector $\mathbf{x}-\mathbf{x}^{\prime}$, or the scalar field in the problem of turbulent advection; the brackets $\langle\ldots\rangle$ denote the ensemble averaging, and the time argument common to all the quantities is omitted in (1.1) and analogous formulas below. The inertial range (or the convective range in the problem of turbulent advection) is defined by the inequalities $l<<r<<L$, where $l \equiv \Lambda^{-1}$ is the internal (viscous) scale and $L \equiv M^{-1}$ is the external (integral) scale.

According to the $\mathrm{KO}$ theory, the functions (1.1) in the inertial range are independent of both the external and internal scales (the First and the Second Kolmogorov hypotheses, respectively) and are determined by the only parameter $\bar{\epsilon}$, the mean dissipation rate, see e.g. [1,2]. Dimensionality considerations then determine the functions (1.1), apart from numerical coefficients, in the form

$$
S_{n}(r)=\operatorname{const}(\bar{\epsilon} r)^{n / 3}
$$

Both experimental and theoretical evidence is known in favor of some deviation from the predictions of the KO theory, see [1, 3] []. For the structure functions (1.1), these deviations are phenomenologically written in the form (in contradiction with the First Kolmogorov hypothesis)

$$
S_{n}(r)=\operatorname{const}(\bar{\epsilon} r)^{n / 3} f_{n}(M r)
$$

where the "scaling functions" $f_{n}(M r)$ are supposed to have power-like behaviour in the asymptotic region $M r<<1$,

$$
f_{n}(M r) \simeq \operatorname{const}(M r)^{q_{n}}
$$

The singular dependence of the structure functions on $M$ for $M \rightarrow 0$ and nonlinearity of the exponents $q_{n}$ in $n$ are usually referred to as "anomalous scaling", and in theoretical models they are explained by strongly developed fluctuations of the local dissipation rate ("intermittency"), see discussion in [1], Chap.25. Within the framework of numerous models, the anomalous exponents $q_{n}$ are related to the statistics of the local dissipation rate or to the 
dimensionality of fractal structures formed by small-scale vortices in the dissipative range, see for a review [7]. As a rule, these models are only loosely related to underlying microscopic models, and therefore some doubt remains about the universality of representations like (1.3), (1.4) and the very existence of deviations from the KO theory.

An effective method of studying self-similar scaling behaviour is that of the renormalization group ( $\mathrm{RG}$ ), see [8.9]. It was successfully applied in the theory of critical phenomena to explain the origin of critical scaling and to calculate universal quantities (critical dimensions and scaling functions) in the form of series in the formal small parameter $\varepsilon=4-d$, where $d$ is the space dimensionality, see [10]. This technique is also fully applicable to the theory of turbulence [11], see also the review paper [12] and references therein.

In the statistical theory of turbulence, the microscopic model is usually taken to be the stochastic Navier-Stokes equation (SNS) with an external random force which imitates the injection of energy by large-scale modes, see e.g. [1.2.12]. The role of the RG expansion parameter is played by the exponent $\varepsilon$ entering into the random force correlator. For the structure functions (1.1), the RG method allows proof of the Second Kolmogorov hypothesis (independence of the viscous length) for a wide variety of realistic random forces, see [12 14]. This is equivalent to the representation (1.3), in which the form of the scaling function $f_{n}(M r)$ is arbitrary. We note that the representations (1.3) for any functions $f_{n}(M r)$ imply the existence of scaling in the infrared (IR) region $L, r>>l$ with definite "critical dimensions"

$$
\Delta_{M}=-\Delta_{r}=1, \quad \Delta\left[S_{n}\right]=-n / 3
$$

for any fixed value of $\bar{\epsilon}$. This means that the structure functions (1.3) scale as $S_{n} \rightarrow \lambda^{\Delta\left[S_{n}\right]} S_{n}$ upon the substitution $M \rightarrow \lambda^{\Delta_{M}} M, r \rightarrow \lambda^{\Delta_{r}} r$ [in general, the exponent $\Delta\left[S_{n}\right]$ is replaced by the critical dimension of the corresponding correlation function; this dimension is calculated within the $\varepsilon$ expansion]. It is this IR scaling that is analogous to the critical scaling in the theory of critical phenomena, where the role of an external scale is played by the correlation length $L \equiv r_{c}$, see [10]. In the RG approach, the critical dimensions like (1.5) arise as coefficients in the RG equations. The form of the scaling functions is not determined by the RG equations themselves, and therefore the anomalous exponents in (1.4) are not related to the critical dimensions of the functions (1.1).

As in the case of critical phenomena [10], the dependence of the scaling functions on the argument $M r$ in the region $M r<<1$ is studied here using the well known operator product expansion (OPE, or $\mathrm{SDE}=$ short distance expansion), see [12-17]. According to the OPE, the scaling functions in expressions like (1.3) are represented in the form

$$
f(M r)=\sum_{F} C_{F}(M r)^{\Delta_{F}}, \quad M r \rightarrow 0 .
$$


Here $C_{F}$ are coefficients regular in $M$, the summation is implied over all possible composite operators $F$ allowed by the symmetry of the left-hand side, and $\Delta_{F}$ are their critical dimensions. In particular, only scalar Galilean invariant operators with nonzero mean value contribute to the OPE for the structure functions (1.1), see [12, 15].

In the theory of critical phenomena, all the nontrivial composite operators have positive critical dimensions, $\Delta_{F}>0$, and the leading term in (1.6) is determined by the simplest operator $F=1$ with $\Delta_{F}=0$, i.e., the function $f(M r)$ is finite as $M \equiv r_{c}^{-1} \rightarrow 0$, see [10]. It has long been realized [13] (see also papers [12,14-17]) that the singular behaviour of the scaling functions in the SNS model for $M \rightarrow 0$ is related to the existence in the model of composite operators with negative critical dimensions. These operators, called "dangerous" in [13], should not be confused with IR relevant operators considered in 18, 19, whose existence can lead to the violation of the scaling regime.

Dangerous composite operators in the SNS model occur only for finite values of the RG expansion parameter $\varepsilon$, and within the $\varepsilon$ expansion it is impossible to decide whether or not a given operator is dangerous, provided its critical dimension is not found exactly using the Schwinger-type functional equations or the Galilean symmetry, see [12,15-17,19-21]. Moreover, dangerous operators enter into the operator product expansions in the form of infinite families with the spectrum of critical dimensions unbounded from below, and the analysis of the small $M$ behaviour implies the summation of their contributions. Such a summation for the case of different-time correlators, first accomplished in [13 (see also [12,15]), establishes the substantial $M$ dependence of the correlators and their superexponential decay as the time differences increase. Of course, this effect is well-known and has a simple physical interpretation as the transport of turbulent eddies as a whole by the large-scale modes, see [22]. Therefore, this effect is not ignored within the correct RG formalism, as it is sometimes believed 23,24, provided the RG is used beyond the $\varepsilon$ expansion and is combined with the OPE technique.

The analysis of the $M$ dependence of Galilean invariant quantities, such as the singletime structure functions (1.1), requires the explicit construction of all dangerous invariant scalar operators, exact calculation of their critical dimensions, and summation of their contributions in the corresponding operator product expansions (11.6). This is clearly not a simple problem and it requires considerable improvement of the present technique.

In view of the difficulties encountered by the RG approach to the SNS model it is tempting to apply the formalism to simpler models, which exhibit some of the features of real turbulent flows, but are easier to study. An interesting example is provided by the wellknown Heisenberg model, see [1], Chap.17, whose exact solution has recently been rederived using the RG method 25]. Unfortunately, the Heisenberg model does not involve higherorder functions and is therefore not suitable for studying anomalous scaling.

Recently, much attention has been attracted by a simple model of the passive advection 
of a scalar quantity by a Gaussian velocity field, introduced by Obukhov [26] and Kraichnan [27], see the papers [28-40] and references therein. It turns out, that the structure functions of the scalar field in this model exhibit anomalous scaling behaviour analogous to (1.3), (1.4), and the corresponding anomalous exponents can be calculated explicitly within expansions in certain small parameters, [31-34].

The advection of a passive scalar field $\theta(x) \equiv \theta(t, \mathbf{x})$ is described by the stochastic equation

$$
\nabla_{t} \theta=\nu_{0} \triangle \theta+f, \quad \nabla_{t} \equiv \partial_{t}+v_{i} \partial_{i},
$$

where $\partial_{t} \equiv \partial / \partial t, \partial_{i} \equiv \partial / \partial x_{i}, \nu_{0}$ is the molecular diffusivity coefficient, $\triangle$ is the Laplace operator, $\mathbf{v}(x)$ is the transverse (owing to the incompressibility) velocity field, and $f \equiv f(x)$ is a Gaussian scalar noise with zero mean and correlator

$$
\left\langle f(x) f\left(x^{\prime}\right)\right\rangle=\delta\left(t-t^{\prime}\right) C(M r), \quad r \equiv\left|\mathbf{x}-\mathbf{x}^{\prime}\right| .
$$

The parameter $L \equiv M^{-1}$ is an integral scale related to the scalar noise, and $C(M r)$ is some function finite as $L \rightarrow \infty$. Without loss of generality, we take $C(0)=1$ (the dimensional coefficient in (1.8) can be absorbed by appropriate rescaling of the field $\theta$ and noise $f$ ).

In a more realistic formulation, the field $\mathbf{v}(x)$ satisfies the stochastic Navier-Stokes equation, see e.g. [41]. Following [26-34], we shall consider a simplified model in which $\mathbf{v}(x)$ obeys a Gaussian distribution with zero average and correlator

$$
\left\langle v_{i}(x) v_{j}\left(x^{\prime}\right)\right\rangle=D_{0} \frac{\delta\left(t-t^{\prime}\right)}{(2 \pi)^{d}} \int d \mathbf{k} P_{i j}(\mathbf{k})\left(k^{2}+m^{2}\right)^{-d / 2-\varepsilon / 2} \exp \left[\mathbf{i k} \cdot\left(\mathbf{x}-\mathbf{x}^{\prime}\right)\right],
$$

where $P_{i j}(\mathbf{k})=\delta_{i j}-k_{i} k_{j} / k^{2}$ is the transverse projector, $k \equiv|\mathbf{k}|, D_{0}$ is an amplitude factor, $1 / m$ is another integral scale, and $d$ is the dimensionality of the $\mathbf{x}$ space; $0<\varepsilon<2$ is a parameter with the real ("Kolmogorov") value $\varepsilon=4 / 3 ; \varepsilon=2-\gamma$ in the notation of [31.32], $\varepsilon=\kappa$ in the notation of [33], and $\varepsilon=\chi$ in the notation of [34]. The relations

$$
g_{0} \equiv D_{0} / \nu_{0} \equiv \Lambda^{\varepsilon}
$$

define the coupling constant $g_{0}$ (expansion parameter in the perturbation theory, see Sec.II) and the characteristic UV momentum scale $\Lambda\left(\Lambda \simeq 1 / r_{d}\right.$ in the notation of [31.32]).

In the model (1.7)-(1.9), the odd multipoint correlation functions of the scalar field vanish, while the even single-time functions satisfy linear partial differential equations. The solution for the pair correlator is obtained explicitly; it shows that the structure function $S_{2}$ is finite for $M, m=0$ [27]. The higher-order correlators are not found explicitly, but their asymptotic behaviour for $M \rightarrow 0$ can be extracted from the analysis of the nontrivial zero modes of the corresponding differential operators in the limit $1 / d \rightarrow 0$ [31,32] or $\varepsilon \rightarrow 0$ 
[33,34]. It was shown that the structure functions are finite for $m=0$, and in the convective range $\Lambda>>1 / r>>M$ they have the form (up to the notation):

$$
S_{2 n}(r) \equiv\left\langle\left[\theta(\mathbf{x})-\theta\left(\mathbf{x}^{\prime}\right)\right]^{2 n}\right\rangle \propto D_{0}^{-n} r^{n(2-\varepsilon)}(M r)^{\Delta_{n}},
$$

with negative anomalous exponents $\Delta_{n}$, whose first terms of the expansion in $1 / d$ [31.32] and $\varepsilon[33,34$ have the form

$$
\Delta_{n}=-2 n(n-1) \varepsilon /(d+2)+O\left(\varepsilon^{2}\right)=-2 n(n-1) \varepsilon / d+O\left(1 / d^{2}\right) .
$$

We note that $\Delta_{n}=-\rho_{2 n}$ in the notation of [33], $\Delta_{2}=-\Delta$ in the notation of [31], and $\Delta_{n}=-\Delta_{2 n}$ in [32].

Another quantity of interest is the local dissipation rate, $E(x)=\nu_{0}\left[\partial_{i} \theta(x) \partial_{i} \theta(x)\right]$. The single-time correlation functions of its powers in the convective range have the form [32, 34]:

$$
\left\langle E^{n}(x) E^{m}\left(x^{\prime}\right)\right\rangle \propto(\Lambda r)^{-\Delta_{n}-\Delta_{m}}(M r)^{\Delta_{n+m}}
$$

where $\Lambda$ is defined in (1.10) and the exponents $\Delta_{n}$ are the same as in Eq.(1.11).

In this paper, we apply the RG and OPE approach developed in [12-17,19-21,41] to the model (1.7)-(1.9). We show that the RG explains the origin of anomalous scaling and allows the anomalous exponents to be calculated in the form of a series in the parameter $\varepsilon$ entering into the correlator (1.8). Therefore, this parameter plays in the RG approach the role analogous to that played by the parameter $\varepsilon=4-d$ in the RG theory of critical phenomena, and the results obtained in [33, 34] can be interpreted as the first terms of the corresponding $\varepsilon$ expansions.

We have calculated the exponents $\Delta_{n}$ in the second order of the $\varepsilon$ expansion for an arbitrary value of the space dimensionality $d$ (they are given in Sec.IIII). In particular, we have obtained

$$
\begin{array}{r}
\Delta_{n}=-\frac{n(n-1) \varepsilon}{2}+\frac{n(n-1) \varepsilon^{2}}{16}[2 n(19-66 \ln (4 / 3))+5-24 \ln (4 / 3)]+O\left(\varepsilon^{3}\right)= \\
=-n(n-1) \varepsilon / 2+\varepsilon^{2} n(n-1)[0.00162 n-0.11902]+O\left(\varepsilon^{3}\right)
\end{array}
$$

for $d=2$ and

$$
\begin{array}{r}
\Delta_{n}=-\frac{2 n(n-1) \varepsilon}{5}+\frac{2 n(n-1) \varepsilon^{2}}{875} \times \\
\times[2 n(255 \pi \sqrt{3}-1384)+345 \pi \sqrt{3}-1884]+O\left(\varepsilon^{3}\right)= \\
=-2 n(n-1) \varepsilon / 5+\varepsilon^{2} n(n-1)[0.01626 n-0.01535]+O\left(\varepsilon^{3}\right)
\end{array}
$$

for $d=3$.

Representations like (1.11), (1.13) can also be derived using the RG and OPE for any correlation function; as an additional example, we consider the single-time pair correlators involving second-rank irreducible tensors of the form 


$$
\partial_{i} \theta \partial_{j} \theta\left[\partial_{s} \theta \partial_{s} \theta\right]^{n-2}-\delta_{i j}\left[\partial_{s} \theta \partial_{s} \theta\right]^{n} / d,
$$

the special case $n=2$ was considered previously in [32].

From the RG viewpoints, the model (11.7)-(1.9) is simpler than the "true" SNS model in at least two respects. First, dangerous operators in the model (1.7)-(1.9) exist even for asymptotically small values of $\varepsilon$ and therefore they can be identified within the $\varepsilon$ expansion. These are the local dissipation rate $E(x)$ and all its powers, their critical dimensions being $\Delta_{n}$. Second, only finite number of these operators contribute to the operator product expansion (1.6) for any given correlation function, and the additional resummation of the series (11.6), discussed above, is not required here: the leading term of the asymptotic behaviour of the function $f(M r)$ for $M r \rightarrow 0$ is simply given by the contribution of the "most dangerous" operator, i.e., that having the smallest value of $\Delta_{n}$.

It was suggested in [33] that the anomalous exponents describing the asymptotic behaviour for $M r<<1$ can be related to certain IR relevant interactions in a hypothetical RG approach, see also discussion in [32]. This is not exactly so: in fact, these exponents in the model (1.7)-(1.9) are related to the critical dimensions of certain "dangerous" composite operators in the operator product expansions for corresponding Green functions. It should be stressed, that in the SNS model the behaviour at $M r \rightarrow 0$ will probably be determined by additional resummations of the operator product expansions, which can lead to more exotic behaviour than the simple power-like one in (11.11), (1.13).

The plan of our paper is the following. In Sec.【1, we discuss the field theoretical formulation and UV renormalization of the model (1.7)-(1.9) and derive the corresponding RG equations with exactly known RG functions (the $\beta$ function and the anomalous dimension). These equations have an IR stable fixed point, which establishes the existence of IR scaling with exactly known critical dimensions of the basic fields and parameters of the model. In Sec.III, we discuss the renormalization of various composite operators in the model (1.7)(1.9). In particular, we present the second-order result for the $\varepsilon$ expansion of the quantity $\Delta_{n}$, the critical dimension associated with the operator $E^{n}(x)$. In Sec IV, it is explained using the OPE that, at the same time, $\Delta_{n}$ play the part of the anomalous exponents in relations like (1.11), (1.13). The results obtained are discussed in Sec.(7. We also briefly mention there one of the possible modifications of the model (1.7)-(1.9), that of a "slow" velocity field, in which the correlator (1.9) contains no $\delta$ function in time. The RG analysis shows that the Green functions in this model exhibit an anomalous scaling behaviour, and the corresponding anomalous exponents are calculated in the form of series in $\varepsilon+2$. 


\section{FIELD THEORETICAL FORMULATION, UV RENORMALIZATION, AND RG EQUATIONS}

The stochastic problem ([1.7)-([1.9) is equivalent to the field theoretical model of the set of three fields $\Phi \equiv\left\{\theta, \theta^{\prime}, \mathbf{v}\right\}$ with action functional

$$
S(\Phi)=\theta^{\prime} D_{\theta} \theta^{\prime} / 2+\theta^{\prime}\left[-\partial_{t} \theta-(\mathbf{v} \boldsymbol{\partial}) \theta+\nu_{0} \triangle \theta\right]-\mathbf{v} D_{v}^{-1} \mathbf{v} / 2
$$

The first four terms in (2.1) represent the Martin-Siggia-Rose-type action [42-45] for the stochastic problem (1.7), (1.8) at fixed $\mathbf{v}$, and the last term represents the Gaussian averaging over v. Here $D_{\theta}$ and $D_{v}$ are the correlators (11.8) and (11.9), respectively, the required integrations over $x=(t, \mathbf{x})$ and summations over the vector indices are understood.

The formulation (2.1) means that statistical averages of random quantities in stochastic

problem (1.7)-(1.9) coincide with functional averages with the weight $\exp S(\Phi)$, therefore generating functionals of total $(G(A))$ and connected $(W(A))$ Green functions of the problem are represented by the functional integral

$$
G(A)=\exp W(A)=\int \mathcal{D} \Phi \exp [S(\Phi)+A \Phi]
$$

with arbitrary sources $A \equiv A^{\theta}, A^{\theta^{\prime}}, A^{\mathbf{v}}$ in the linear form

$$
A \Phi \equiv \int d x\left[A^{\theta}(x) \theta(x)+A^{\theta^{\prime}}(x) \theta^{\prime}(x)+A_{i}^{\mathbf{v}}(x) v_{i}(x)\right]
$$

The model (2.1) corresponds to a standard Feynman diagrammatic technique with the triple vertex $-\theta^{\prime}(\mathbf{v} \boldsymbol{\partial}) \theta$ and bare propagators (in the momentum-frequency representation)

$$
\begin{array}{r}
\left\langle\theta \theta^{\prime}\right\rangle_{0}=\left\langle\theta^{\prime} \theta\right\rangle_{0}^{*}=\left(-i \omega+\nu_{0} k^{2}\right)^{-1}, \\
\langle\theta \theta\rangle_{0}=C(k)\left(\omega^{2}+\nu_{0}^{2} k^{4}\right)^{-1} \\
\left\langle\theta^{\prime} \theta^{\prime}\right\rangle_{0}=0
\end{array}
$$

where $C(k)$ is the Fourier transform of the function $C(M r)$ in (1.8) and the bare propagator $\langle\mathbf{v v}\rangle_{0}$ is given by Eq.(1.9). The role of the coupling constant in the perturbation theory is played by the parameter $g_{0}$ defined in (1.10).

It is well known that the analysis of UV divergences is based on the analysis of canonical dimensions. Dynamical models of the type (2.1), in contrast to static models, are two-scale, i.e., to each quantity $F$ (a field or a parameter in the action functional) one can assign two independent canonical dimensions, the momentum dimension $d_{F}^{k}$ and the frequency dimension $d_{F}^{\omega}$, determined from the natural normalization conditions

$$
d_{k}^{k}=-d_{\mathbf{x}}^{k}=1, d_{k}^{\omega}=d_{\mathbf{x}}^{\omega}=0, d_{\omega}^{k}=d_{t}^{k}=0, d_{\omega}^{\omega}=-d_{t}^{\omega}=1
$$


and from the requirement that each term of the action functional be dimensionless (with respect to the momentum and frequency dimensions separately), see [12,20. Then, based on $d_{F}^{k}$ and $d_{F}^{\omega}$, one can introduce the "summed" (total) canonical dimension $d_{F}=d_{F}^{k}+2 d_{F}^{\omega}$ (in the free theory, $\partial_{t} \propto \triangle$ ).

The dimensions for the model (2.1) are given in Table \&, including renormalized parameters, which will be considered later on.

From Table \it follows that the model is logarithmic (the coupling constant $g_{0}$ is dimensionless) at $\varepsilon=0$, and the UV divergences have the form of the poles in $\varepsilon$ in the Green functions.

The total dimension $d_{F}$ plays in the theory of renormalization of dynamical models the same role as does the conventional (momentum) dimension in static problems. The canonical dimensions of an arbitrary 1-irreducible Green function $\Gamma=\langle\Phi \ldots \Phi\rangle_{1-\text { ir }}$ are given by the relations

$$
\begin{array}{r}
d_{\Gamma}^{k}=d-N_{\Phi} d_{\Phi}, \\
d_{\Gamma}^{\omega}=1-N_{\Phi} d_{\Phi}^{\omega}, \\
d_{\Gamma}=d_{\Gamma}^{k}+2 d_{\Gamma}^{\omega}=d+2-N_{\Phi} d_{\Phi},
\end{array}
$$

where $N_{\Phi}=\left\{N_{\theta}, N_{\theta^{\prime}}, N_{\mathbf{v}}\right\}$ are the numbers of corresponding fields entering into the function $\Gamma$, and the summation over all types of the fields is implied. The total dimension $d_{\Gamma}$ is the formal index of the UV divergence. Superficial UV divergences, whose removal requires counterterms, can be present only in those functions $\Gamma$ for which $d_{\Gamma}$ is a nonnegative integer, see e.g. [8,9].

Analysis of divergences should be based on the following auxiliary considerations:

(1) From the explicit form of the vertex and bare propagators in the model (2.1) it follows that $N_{\theta^{\prime}}-N_{\theta}=2 N_{0}$ for any 1-irreducible Green function, where $N_{0} \geq 0$ is the total number of the bare propagators $\langle\theta \theta\rangle_{0}$ entering into the function (obviously, no diagrams with $N_{0}<0$ can be constructed). Therefore, the difference $N_{\theta^{\prime}}-N_{\theta}$ is an even nonnegative integer for any nonvanishing function.

(2) If for some reason a number of external momenta occur as an overall factor in all the diagrams of a given Green function, the real index of divergence $d_{\Gamma}^{\prime}$ is smaller than $d_{\Gamma}$ by the corresponding number of unities (the Green function requires counterterms only if $d_{\Gamma}^{\prime}$ is a nonnegative integer).

In the model (2.1), the derivative $\partial$ at the vertex $\theta^{\prime}(\mathbf{v} \boldsymbol{\partial}) \theta$ can be moved onto the field $\theta^{\prime}$ by virtue of the transversality of the field $\mathbf{v}$. Therefore, in any 1-irreducible diagram it is always possible to move the derivative onto any of the external "tails" $\theta$ or $\theta^{\prime}$, which decreases the real index of divergence: $d_{\Gamma}^{\prime}=d_{\Gamma}-N_{\theta}-N_{\theta^{\prime}}$. The fields $\theta, \theta^{\prime}$ enter into the counterterms only in the form of derivatives $\partial \theta, \partial \theta^{\prime}$. 
From the dimensions in Table [ [ we find $d_{\Gamma}=d+2-N_{\mathbf{v}}+N_{\theta}-(d+1) N_{\theta^{\prime}}$ and $d_{\Gamma}^{\prime}=(d+2)\left(1-N_{\theta^{\prime}}\right)-N_{\mathbf{v}}$. From these expressions it follows that for any $d$, superficial divergences can exist only in the 1-irreducible functions $\left\langle\theta^{\prime} \theta \ldots \theta\right\rangle$ with $N_{\theta^{\prime}}=1$ and arbitrary value of $N_{\theta}$, for which $d_{\Gamma}=2, d_{\Gamma}^{\prime}=0$. However, all the functions with $N_{\theta}>N_{\theta^{\prime}}$ vanish (see above) and obviously do not require counterterms. We are left with the only superficially divergent function $\left\langle\theta^{\prime} \theta\right\rangle$; the corresponding counterterm must contain two symbols $\partial$ and is therefore reduced to $\theta^{\prime} \triangle \theta$. Inclusion of this counterterm is reproduced by the multiplicative renormalization of the parameters $g_{0}, \nu_{0}$ in the action functional (2.1) with the only independent renormalization constant $Z_{\nu}$ :

$$
\nu_{0}=\nu Z_{\nu}, \quad g_{0}=g \mu^{\varepsilon} Z_{g}, \quad Z_{g}=Z_{\nu}^{-1} .
$$

Here $\mu$ is the renormalization mass in the minimal subtraction scheme (MS), which we always use in what follows, $g$ and $\nu$ are renormalized analogues of the bare parameters $g_{0}$ and $\nu_{0}$, and $Z=Z(g, \varepsilon, d)$ are the renormalization constants. Their relation in (2.5) results from the absence of renormalization of the contribution with $D_{0}$ in (2.1), so that $D_{0} \equiv g_{0} \nu_{0}=g \mu^{\varepsilon} \nu$, see (1.10). No renormalization of the fields and "masses" is required, i.e., $Z_{\Phi}=1$ for all $\Phi$ and $m_{0}=m, M_{0}=M, Z_{m}=Z_{M}=1$.

Since the fields are not renormalized, their renormalized Green functions $W^{R}$ coincide with the corresponding unrenormalized functions $W=\langle\Phi \ldots \Phi\rangle$ (for definiteness, we discuss the connected functions); the only difference is in the choice of variables and in the form of perturbation theory (in $g$ instead of $g_{0}$ ):

$$
W^{R}(g, \nu, \mu, \ldots)=W\left(g_{0}, \nu_{0}, \ldots\right)
$$

(the dots stand for other arguments like coordinates and momenta). We use $\widetilde{\mathcal{D}}_{\mu}$ to denote the differential operator $\mu \partial_{\mu}$ for fixed bare parameters $g_{0}, \nu_{0}$ and operate on both sides of Eq.(2.6) with it. This gives the basic differential RG equation:

$$
\begin{array}{r}
\mathcal{D}_{R G} W^{R}(g, \nu, \mu, \ldots)=0 \\
\mathcal{D}_{R G} \equiv \mathcal{D}_{\mu}+\beta(g) \partial_{g}-\gamma_{\nu}(g) \mathcal{D}_{\nu}
\end{array}
$$

where we have written $\mathcal{D}_{s} \equiv s \partial_{s}$ for any variable $s$, and the RG functions (the $\beta$ function and the anomalous dimension $\gamma$ ) are defined as

$$
\gamma_{\nu}(g) \equiv \widetilde{\mathcal{D}}_{\mu} \ln Z_{\nu}, \quad \beta(g) \equiv \widetilde{\mathcal{D}}_{\mu} g=g\left[-\varepsilon+\gamma_{\nu}\right]
$$

The relation between $\beta$ and $\gamma$ results from the definitions and the last relation in (2.5). In general, if some quantity $G$ is renormalized multiplicatively, $G=Z_{G} G^{R}$, it satisfies the RG equation of the form 


$$
\left[\mathcal{D}_{R G}+\gamma_{G}(g)\right] G^{R}=0, \quad \gamma_{G}(g) \equiv \widetilde{\mathcal{D}}_{\mu} \ln Z_{G}
$$

with the operator $\mathcal{D}_{R G}$ from (2.7).

Explicit calculation of the constant $Z_{\nu}$ in the model (2.1) in the one-loop approximation gives:

$$
Z_{\nu}=1-\frac{g(d-1) C_{d}}{2 d \varepsilon},
$$

where we have written $C_{d} \equiv S_{d} /(2 \pi)^{d}$ and $S_{d} \equiv 2 \pi^{d / 2} / \Gamma(d / 2)$ is the surface area of the unit sphere in $d$-dimensional space.

The one-loop approximation (2.10) for the constant $Z_{\nu}$ is in fact exact, i.e., it has no corrections of order $g^{2}, g^{3}$, and so on. Indeed, from the explicit form of the vertex and the bare propagators (1.9), (2.3) it follows that any multiloop diagram of the 1-irreducible function $\left\langle\theta^{\prime} \theta\right\rangle$ contains effectively a closed circuit of retarded propagators $\left\langle\theta \theta^{\prime}\right\rangle_{0}$ and therefore vanishes (it is also important here that the propagator $\langle\mathbf{v v}\rangle_{0}$ in (1.9) is proportional to the $\delta$ function in time).

From the definitions (2.8) using Eq.(2.10) we find exact expressions for the basic RG functions:

$$
\gamma_{\nu}(g)=\frac{g(d-1) C_{d}}{2 d}, \quad \beta(g)=g\left[-\varepsilon+\frac{g(d-1) C_{d}}{2 d}\right] .
$$

From (2.11) it follows that an IR-attractive fixed point

$$
g_{*}=\frac{2 d \varepsilon}{C_{d}(d-1)}
$$

of the RG equations $\left[\beta\left(g_{*}\right)=0, \beta^{\prime}\left(g_{*}\right)=\varepsilon>0\right.$ ] exists in the physical region $g>0$ for all $\varepsilon>0$. The value of $\gamma_{\nu}(g)$ at the fixed point is also found exactly:

$$
\gamma_{\nu}^{*} \equiv \gamma_{\nu}\left(g_{*}\right)=\varepsilon,
$$

without corrections of order $\varepsilon^{2}, \varepsilon^{3}$, and so on.

It is well known that the leading term of the IR asymptotic expression of any renormalized quantity $G^{R}$, for which the RG equation of the form (2.9) is valid, satisfies the same equation with the substitution $g \rightarrow g_{*}$, where $g_{*}$ is the IR stable fixed point:

$$
\left[\mathcal{D}_{\mu}-\gamma_{\nu}^{*} \mathcal{D}_{\nu}+\gamma_{G}^{*}\right] G^{R}=0, \quad \gamma_{G}^{*} \equiv \gamma_{G}^{*}\left(g_{*}\right) .
$$

Canonical scale invariance is expressed by the relations:

$$
\left[\sum_{\alpha} d_{\alpha}^{k} \mathcal{D}_{\alpha}-d_{G}^{k}\right] G^{R}=0, \quad\left[\sum_{\alpha} d_{\alpha}^{\omega} \mathcal{D}_{\alpha}-d_{G}^{\omega}\right] G^{R}=0,
$$


in which $\alpha \equiv\{t, \mathbf{x}, \mu, \nu, m, M, g\}$ is the set of all arguments of $G^{R}(t, \mathbf{x}$ is the set of all times and coordinates), and $d^{k}$ and $d^{\omega}$ are the canonical dimensions of $G^{R}$ and $\alpha$. Substituting the needed dimensions from Table [ into (2.15), we obtain:

$$
\begin{aligned}
{\left[\mathcal{D}_{\mu}+\mathcal{D}_{m}+\mathcal{D}_{M}-2 \mathcal{D}_{\nu}-\mathcal{D}_{\mathrm{x}}-d_{G}^{k}\right] G^{R} } & =0, \\
{\left[\mathcal{D}_{\nu}-\mathcal{D}_{t}-d_{G}^{\omega}\right] G^{R} } & =0 .
\end{aligned}
$$

Each of the equations (2.14)-(2.16) describes scaling with dilatation of all variables, the derivatives with respect to which enter into the differential operator. We are interested in scaling with dilatation of $t, \mathbf{x}$, and "masses" $M, m$ for fixed $\mu, \nu$, and $g$, and it is necessary to exclude the corresponding derivatives $\mathcal{D}_{\alpha}$ by a combination of the available equations. After eliminating $\mathcal{D}_{\mu}$ and $\mathcal{D}_{\nu}$ from (2.14) and (2.16) we obtain the desired equation of critical IR scaling for the model (2.1):

$$
\left[-\mathcal{D}_{\mathbf{x}}+\Delta_{t} \mathcal{D}_{t}+\Delta_{m} \mathcal{D}_{m}+\Delta_{M} \mathcal{D}_{M}-\Delta_{G}\right] G^{R}=0
$$

with the coefficients

$$
\Delta_{t}=-\Delta_{\omega}=-2+\gamma_{\nu}^{*}=-2+\varepsilon, \quad \Delta_{m}=\Delta_{M}=1
$$

and

$$
\Delta[G] \equiv \Delta_{G}=d_{G}^{k}+\Delta_{\omega} d_{G}^{\omega}+\gamma_{G}^{*},
$$

which are the corresponding critical dimensions. In particular, for any correlation function $G^{R}=W^{R}=\langle\Phi \ldots \Phi\rangle$ of the fields $\Phi$ we have $\Delta_{G}=N_{\Phi} \Delta_{\Phi}$, with the summation over all fields $\Phi$ entering into $G^{R}$, and for $\Delta_{\Phi}$ using the data from Table 1 and the exact value of $\gamma_{\nu}^{*}=\varepsilon$ we obtain from (2.19) the following exact expressions:

$$
\Delta_{\mathbf{v}}=1-\varepsilon, \quad \Delta_{\theta}=-1+\varepsilon / 2, \quad \Delta_{\theta^{\prime}}=d+1-\varepsilon / 2
$$

(we recall that the fields in the model (2.1) are not renormalized and therefore $\gamma_{\Phi}=0$ for all $\Phi)$.

To avoid misunderstanding, we again emphasize the fact that the RG equation (2.17) describes IR scaling, i.e., the statement which is equivalent to the critical scaling in the theory of critical phenomena, see also discussion in [12,13]. In this scaling the variables $M$, $m$ are also IR relevant, i.e., they are dilated in scale transformations. In other words, the expression (2.17) describes the asymptotic behaviour as $\Lambda r>>1$ for any fixed values of $M r$ and $m r$, with the UV scale $\Lambda$ defined in (11.10). The solution of the set of equations (2.16), (2.17) can be found only up to some unknown function of all the first integrals, including those of the form $m r, M r$, where $r=\left|\mathbf{x}_{i}-\mathbf{x}_{j}\right|$ is some coordinate difference. 
The anomalous exponents in the expressions (1.11), (1.13) describe the behaviour of the corresponding correlation functions for $M r \rightarrow 0$, and therefore are not related to the their critical dimensions (2.19).

In terms of unrenormalized variables, the solution of the set of equations (2.15), (2.17) for the example of a single-time pair correlation function can be found, apart from numerical factor, in the form (for more details, see e.g. [12):

$$
G \propto G^{R} \simeq D_{0}^{d_{G}^{\omega}} \Lambda^{-\gamma_{G}^{*}} r^{-\Delta_{G}} f(M r, m r),
$$

with certain, as yet unknown, scaling function $f$.

In what follows we limit ourselves to the correlation functions of the form (1.11), (1.13), which are finite for $m=0$, see [31-34]. We shall always set $m=0$ and study the dependence

of the scaling functions $f(M r) \equiv f(M r, m r=0)$ on the only remaining argument $M r$ in the asymptotic region $M r<<1$. This can be performed within the framework of the general solution (2.21) of the RG equations with the aid of additional methods, see Sec.IV.

\section{RENORMALIZATION AND CRITICAL DIMENSIONS OF COMPOSITE OPERATORS}

The quantities entering into the left hand sides of Eqs.(1.11), (1.13) are two-point correlation functions of composite fields (composite operators in quantum-field terminology) rather than multipoint correlators of the primary fields. In what follows, we use the term "composite operator" for any local (unless stated to be otherwise) monomial or polynomial constructed from primary fields and their derivatives at a single point $x \equiv(t, \mathbf{x})$. Examples are $\theta^{n}(x),\left[\partial_{i} \theta(x) \partial_{i} \theta(x)\right]^{n}, \partial_{i} \theta(x) \partial_{j} \theta(x), \theta^{\prime}(x) \nabla_{t} \theta(x)$ and so on.

Since the arguments of the fields coincide, correlation functions with these operators contain additional UV divergences, which are removed by additional renormalization procedure, see e.g. [9]. For the renormalized correlation functions standard RG equations are obtained, which describe IR scaling with definite critical dimensions $\Delta_{F} \equiv \Delta[F]$ of certain "basis" operators $F$. Owing to the renormalization, $\Delta[F]$ does not coincide in general with the naïve sum of critical dimensions of the fields and derivatives entering into $F$.

Detailed exposition of the renormalization of composite operators for the stochastic Navier-Stokes equation is given in the review paper [12], see also [13-17,19-21], below we confine ourselves to only the necessary information.

In general, composite operators are mixed in renormalization, i.e., an UV finite renormalized operator $F^{R}$ has the form $F^{R}=F+$ counterterms, where the contribution of the counterterms is a linear combination of $F$ itself and, possibly, other unrenormalized operators which "admix" to $F$ in renormalization. 
Let $F \equiv\left\{F_{\alpha}\right\}$ be a closed set, all of whose monomials mix only with each other in renormalization. The renormalization matrix $Z_{F} \equiv\left\{Z_{\alpha \beta}\right\}$ and the matrix of anomalous dimensions $\gamma_{F} \equiv\left\{\gamma_{\alpha \beta}\right\}$ for this set are given by

$$
F_{\alpha}=\sum_{\beta} Z_{\alpha \beta} F_{\beta}^{R}, \quad \gamma_{F}=Z_{F}^{-1} \widetilde{\mathcal{D}}_{\mu} Z_{F},
$$

and the corresponding matrix of critical dimensions $\Delta_{F} \equiv\left\{\Delta_{\alpha \beta}\right\}$ is given by Eq.(2.19), in which $d_{F}^{k}, d_{F}^{\omega}$, and $d_{F}$ are understood as the diagonal matrices of canonical dimensions of the operators in question (with the diagonal elements equal to sums of corresponding dimensions of all fields and derivatives constituting $F$ ) and $\gamma^{*} \equiv \gamma\left(g_{*}\right)$ is the matrix (3.1) at the fixed point.

Critical dimensions of the set $F \equiv\left\{F_{\alpha}\right\}$ are given by the eigenvalues of the matrix $\Delta_{F}$. The "basis" operators that possess definite critical dimensions have the form

$$
\bar{F}_{\alpha}^{R}=\sum_{\beta} U_{\alpha \beta} F_{\beta}^{R}
$$

where the matrix $U_{F}=\left\{U_{\alpha \beta}\right\}$ is such that $\Delta_{F}^{\prime}=U_{F} \Delta_{F} U_{F}^{-1}$ is diagonal.

In general, counterterms to a given operator $F$ are determined by all possible 1irreducible Green functions with one operator $F$ and arbitrary number of primary fields, $\Gamma=\left\langle F(x) \Phi\left(x_{1}\right) \ldots \Phi\left(x_{2}\right)\right\rangle$. The total canonical dimension (formal index of divergence) for such functions is given by

$$
d_{\Gamma}=d_{F}-N_{\Phi} d_{\Phi}
$$

with the summation over all types of fields entering into the function. For superficially divergent diagrams, $d_{\Gamma}$ is a nonnegative integer, cf. Sec.П!

Let us consider operators of the form $\theta^{n}(x)$ with the canonical dimension $d_{F}=-n$, entering into the structure functions (1.11). From Table [in Sec.I1 and Eq.(3.3) we obtain $d_{\Gamma}=-n+N_{\theta}-N_{\mathbf{v}}-(d+1) N_{\theta^{\prime}}$, and from the analysis of the diagrams it follows that the total number of the fields $\theta$ entering into the function $\Gamma$ can never exceed the number of the fields $\theta$ in the operator $\theta^{n}$ itself, i.e., $N_{\theta} \leq n$. Therefore, the divergence can only exist in the functions with $N_{\mathbf{v}}=0, N_{\theta^{\prime}}=0$, and arbitrary value of $n=N_{\theta}$, for which the formal index vanishes, $d_{\Gamma}=0$. However, at least one of $N_{\theta}$ external "tails" of the field $\theta$ is attached to a vertex $\theta^{\prime}(\mathbf{v} \boldsymbol{\partial}) \theta$ (it is impossible to construct nontrivial, superficially divergent diagram of the desired type with all the external tails attached to the vertex $F$ ), at least one derivative $\partial$ appears as an extra factor in the diagram, and, consequently, the real index of divergence is necessarily negative, see Sec. [1].

This means that the operator $\theta^{n}$ requires no counterterms at all, i.e., it is in fact UV finite, $\theta^{n}=Z\left[\theta^{n}\right]^{R}$ with $Z=1$. It then follows that the critical dimension of $\theta^{n}(x)$ is simply 
given by the expression (2.19) with no correction from $\gamma_{F}^{*}$ and is therefore reduced to the sum of the critical dimensions of the factors:

$$
\Delta\left[\theta^{n}\right]=n \Delta[\theta]=n(-1+\varepsilon / 2) .
$$

We note that this relation was not clear a priori and it is a specific feature of the model (2.1). For example, in the standard model $\phi^{4}$ of the theory of critical phenomena, the critical dimensions of the field $\phi$ and the composite operators $\phi^{2}$ and $\phi^{4}$ are completely independent, and they determine independent critical exponents $\eta, \nu$, and $\omega$, see e.g. [10]. The relation analogous to (3.4) is valid for the powers of the velocity field of the stochastic Navier-Stokes equation, where it is a consequence of the Galilean symmetry of the model, see e.g. [12, 13, 115].

Now let us turn to the operators $F_{n} \equiv\left[\partial_{i} \theta \partial_{i} \theta\right]^{n}$ with $d_{F}=0, d_{F}^{\omega}=-n$. They enter into the left-hand sides of Eqs.(1.13) and, as we shall see in Sec.[V], it is their critical dimensions that determine the anomalous exponents in (1.11) and (1.13).

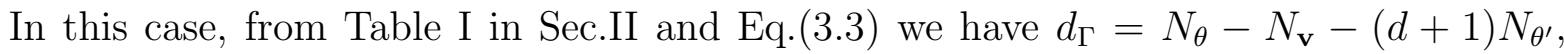
with the necessary condition $N_{\theta} \leq 2 n$, which follows from the structure of the diagrams. It is also obvious from the analysis of the diagrams that the counterterms to these operators can involve the fields $\theta, \theta^{\prime}$ only in the form of derivatives, $\partial \theta, \partial \theta^{\prime}$, and so the real index of divergence has the form $d_{\Gamma}^{\prime}=d_{\Gamma}-N_{\theta}-N_{\theta^{\prime}}=-N_{\mathbf{v}}-(d+2) N_{\theta^{\prime}}$. It then follows that superficial divergences exist only in the Green functions with $N_{\mathbf{v}}=N_{\theta^{\prime}}=0$ and any $N_{\theta} \leq 2 n$, and the corresponding operator counterterms are reduced to the form $F_{k}$ with $k \leq n$. Therefore, the operators $F_{n}$ can mix only with each other in renormalization, the corresponding infinite renormalization matrix $Z_{F}=\left\{Z_{n k}\right\}$ is in fact triangular, $Z_{n k}=0$ for $k>n$, and the critical dimensions associated with the operators $F_{n}$ are determined by the diagonal elements $Z_{n} \equiv Z_{n n}$ (in contradistinction with the case of operators $\theta^{n}$, they are not equal to unity here).

Explicit calculation of the constants $Z_{n}$ in the MS scheme in the two-loop approximation gives:

$$
\begin{array}{r}
Z_{n}^{-1}=1-\frac{u}{2 \varepsilon} \cdot \frac{n(d-1)(d+2 n)}{d(d+2)}+ \\
+\frac{u^{2}}{8 \varepsilon^{2}} \cdot \frac{n(n-1)(d-1)^{2}(d+2 n)(d+2 n+2)}{d^{2}(d+2)^{2}}+ \\
+\frac{u^{2}}{2 \varepsilon} \cdot \frac{n(n-1)(d-1)}{d^{2}(d+2)^{2}(d+4)}\left[\frac{-(d+4)(d+1)}{(d+2)}+\right. \\
\left.+3(d-1)(d+2 n) h_{1}(d) / 4-\frac{(d+1)(d+3 n-2)}{(d+4)} h_{2}(d)\right]+O\left(u^{3}\right),
\end{array}
$$

where we have written $u \equiv g C_{d}$ with the coefficient $C_{d}$ from (2.10), and 


$$
\begin{array}{r}
h_{1}(d) \equiv F(1,1, d / 2+2,1 / 4), \\
h_{2}(d) \equiv F(1,1, d / 2+3,1 / 4)=h_{1}(d+2),
\end{array}
$$

where $F(\ldots)$ is the hypergeometric series (see e.g. [46]):

$$
F(a, b, c, z) \equiv 1+\frac{a b}{c} z+\frac{a(a+1) b(b+1)}{c(c+1)} \cdot \frac{z^{2}}{2 !}+\ldots
$$

From (3.6) and (3.7) it follows:

$$
h_{1}(d)=\sum_{k=0}^{\infty} \frac{k ! \Gamma(d / 2+2)}{4^{k} \Gamma(d / 2+2+k)}, \quad h_{2}(d)=h_{1}(d+2) .
$$

Representations (3.8) are convenient for numerical computations of the functions $h_{1,2}(d)$.

From (3.5) for the anomalous dimension $\gamma_{n} \equiv \widetilde{\mathcal{D}}_{\mu} \ln Z_{n}$ in the two-loop order we obtain:

$$
\begin{array}{r}
\gamma_{n}(g)=-\frac{u n(d-1)(d+2 n)}{2 d(d+2)}+\frac{u^{2} n(n-1)}{d(d+2)(d+4)} \times \\
\times\left[\frac{(d+4)\left(1-d^{2}\right)}{d(d+2)^{2}}+\frac{3(d-1)^{2}(d+2 n)}{4 d(d+2)} h_{1}(d)-\frac{\left(d^{2}-1\right)(d+3 n-2)}{d(d+2)(d+4)} h_{2}(d)\right]+O\left(u^{3}\right),
\end{array}
$$

and for the corresponding critical dimension $\Delta_{n}$ at the fixed point (2.12) using (2.19) and $d_{F}\left[F_{n}\right]=0, d_{F}^{\omega}\left[F_{n}\right]=-n$ (see Table 【 in Sec.【I) we have:

$$
\begin{array}{r}
\Delta_{n}=n \varepsilon+\gamma_{n}^{*}=-\frac{2 n(n-1) \varepsilon}{d+2}+\frac{\varepsilon^{2} n(n-1)}{(d-1)(d+2)^{3}(d+4)^{2}} \times \\
\times\left[-4(d+1)(d+4)^{2}+3(d-1)(d+2)(d+4)(d+2 n) h_{1}(d)-\right. \\
\left.-4(d+1)(d+2)(d+3 n-2) h_{2}(d)\right]+O\left(\varepsilon^{3}\right) .
\end{array}
$$

Expression (3.10) is simplified for any integer value of $d$ owing to the fact that the series in (3.7) reduce to finite sums, see [46]:

$$
h_{1}(d)=2(d+2)\left[(-3)^{d / 2} \ln (4 / 3)+\sum_{k=2}^{d / 2+1} \frac{(-3)^{k-2}}{d / 2-k+2}\right]
$$

for any even value of $d$ and

$$
h_{1}(d)=2(d+2)\left[(-1)^{(d+1) / 2} \cdot 3^{d / 2-1} \cdot \pi+2 \sum_{k=1}^{(d+1) / 2} \frac{(-3)^{(d+1) / 2-k}}{2 k-1}\right]
$$

for any odd value of $d$, which for $d=2$ and $d=3$ gives the results announced in (1.14), (1.15).

We explain in Sec. IV that the critical dimensions $\Delta_{n}$ from (3.10) are nothing else than the anomalous exponents entering into relations (1.11), (1.13), and here we only note that 
the first order in $\varepsilon$ of the expression (3.10) coincides (up to the notation) with the result obtained in [33] for $n=2$ and in [34] for arbitrary $n$, and that the first term of the expansion in $1 / d$ of the expression (3.10) coincides with the result (1.12) obtained in 31,32. We also note that the $\varepsilon^{2}$ term of the expression (3.10) behaves as $1 / d^{2}$ for $1 / d \rightarrow 0$ and therefore gives no contribution to the first order of the $1 / d$ expansion [this follows from the relation $h_{2}(d)=h_{1}(d+2)=1+O(1 / d)$, which is obvious from (3.8)]. This fact suggests that the $\varepsilon$ expansion for the dimension $\Delta_{n}$ in the model (2.1) is "better" than the $1 / d$ expansion in the sense that a given order of the $1 / d$ expansion is contained completely in the corresponding order of the $\varepsilon$ expansion, but not the reverse. We also note that the $n$ dependence of the quantity $\Delta_{n}$ in the second order of the $\varepsilon$ expansion is no longer reduced to the simple factor $n(n-1)$.

The result $\Delta_{1}=0$ in (3.10) is in fact exact, in agreement with the exact solution for the two-point structure function obtained in [27]. Within the RG approach this can be demonstrated using the Schwinger equation of the form

$$
\int \mathcal{D} \Phi \delta\left[\theta(x) \exp S_{R}(\Phi)+A \Phi\right] / \delta \theta^{\prime}(x)=0
$$

(in the general sense of the term, Schwinger equations are any relations stating that any functional integral of a total variational derivative is equal to zero, see e.g. Sec.7 of [47]). In (3.13), $S_{R}$ is the renormalized analog of the action (2.1), and the notation introduced in (2.2) is used. Eq.(3.13) can be rewritten in the form

$$
\left\langle\left\langle\theta^{\prime} D_{\theta} \theta-\nabla_{t}\left[\theta^{2} / 2\right]+\nu Z_{\nu} \triangle\left[\theta^{2} / 2\right]-\nu Z_{\nu} F_{1}\right\rangle\right\rangle_{A}=-A_{\theta^{\prime}} \delta W_{R}(A) / \delta A_{\theta} .
$$

Here $D_{\theta}$ is the correlator (1.8), $\langle\langle\ldots\rangle\rangle_{A}$ denotes the averaging with the weight $\exp \left[S_{R}(\Phi)+\right.$ $A \Phi], W_{R}$ is determined by the Eq.(2.2) with the replacement $S \rightarrow S_{R}$, and the argument $x$ common to all the quantities in (3.14) is omitted.

The quantity $\langle\langle F\rangle\rangle_{A}$ is the generating functional of the correlation functions with one operator $F$ and any number of fields $\Phi$, therefore the UV finiteness of the operator $F$ is equivalent to the finiteness of the functional $\langle\langle F\rangle\rangle_{A}$. The quantity in the right hand side of Eq.(3.14) is finite (a derivative of the renormalized functional with respect to finite argument), and so is the operator in the left hand side. Our operator $F_{1}$ does not admix in renormalization to the operator $\theta^{\prime} D_{\theta} \theta$ ( $F_{1}$ contains too many fields $\theta$ ), and to the operators $\nabla_{t}\left[\theta^{2} / 2\right]$ and $\triangle\left[\theta^{2} / 2\right]$ (they have the form of total derivatives, and $F_{1}$ is not reduced to this form). On the other hand, the operator $\theta^{\prime} D_{\theta} \theta$ does not admix to $F_{1}$ (it is nonlocal, and $F_{1}$ is local), while the derivatives $\nabla_{t}\left[\theta^{2} / 2\right]$ and $\triangle\left[\theta^{2} / 2\right]$ do not admix to $F_{1}$ owing to the fact that each field $\theta$ enters in the counterterms of the operators $F_{n}$ only in the form of derivative $\partial \theta$ (see above). Therefore, all three types of operators entering into the left hand side of the Eq.(3.14) are independent, and they must be UV finite separately. 
Since the operator $\nu Z_{\nu} F_{1}$ is UV finite, it coincides with its finite part, i.e., $\nu Z_{\nu} F_{1}=\nu F_{1}^{R}$, which along with the relation $F_{1}=Z_{1} F_{1}^{R}$ gives $Z_{1}=Z_{\nu}^{-1}$ and therefore $\gamma_{1}=-\gamma_{\nu}$. For the critical exponent $\Delta_{1}=\varepsilon+\gamma_{1}^{*}$ we then obtain $\Delta_{1}=0$ exactly (we recall that $\gamma_{\nu}^{*}=\varepsilon$, see Sec.[I]).

In the SNS model, critical dimensions of certain composite operators can sometimes be obtained exactly using various Schwinger equations and Ward identities for Galilean transformations, see [12-17] and [19-21]. In particular, exact critical dimension of the energy dissipation rate $E(x)$ was found in [20], see also [21]. The simple relation $\Delta\left[E^{n}\right]=n \Delta[E]$ for the powers of the dissipation rate was proposed in [48. It was explained later in [17], that this relation can not be considered reliable, and here we only note that even for the simple Obukhov-Kraichnan model, the critical dimension of $E^{n}$ is not a linear function in $n$, see (3.10), in contradistinction with the powers of the field itself, see (3.4) and [12, 13, 15. for the case of the SNS model.

Critical dimensions of various tensor operators of the form $\partial_{i_{1}} \theta(x) \ldots \partial_{i_{n}} \theta(x)$ can also be calculated in the second order of the $\varepsilon$ expansion from the same two-loop diagrams, which determine the constants (3.5); only the symmetry coefficients differ from those for scalar operators. We shall confine ourselves to the second-rank irreducible traceless tensors of the form

$$
F_{i j}^{n} \equiv \partial_{i} \theta \partial_{j} \theta F_{n-1}-\delta_{i j} F_{n} / d
$$

where $F_{n}$ are the scalar operators discussed above. The operators (3.15) mix only with each other in renormalization, the corresponding renormalization matrix is triangular and its diagonal elements determine the corresponding critical dimensions $\Delta_{n}^{\prime}$. In the second order of the $\varepsilon$ expansion we have obtained:

$$
\begin{array}{r}
\Delta_{n}^{\prime}=\frac{\varepsilon[d(d+1)-2 n(n-1)(d-1)]}{(d-1)(d+2)}+\frac{\varepsilon^{2} n(n-1)}{(d-1)(d+2)^{3}(d+4)^{2}} \times \\
\times\left[-4(d+1)(d+4)^{2} P_{0}+3(d-1)(d+2)(d+4)(d+2 n) h_{1}(d) P_{1}-\right. \\
\left.-4(d+1)(d+2)(d+3 n-2) h_{2}(d) P_{2}\right]+O\left(\varepsilon^{3}\right),
\end{array}
$$

where we have written:

$$
\begin{array}{r}
P_{0}=1+\frac{d}{2 n(n-1)(d-1)}, \\
P_{1}=1-\frac{d(d+1)}{n(d-1)(d+2 n)}, \\
P_{2}=1-\frac{d(2 d-1)}{2 n(d-1)(d+3 n-2)},
\end{array}
$$

and the functions $h_{1,2}(d)$ are defined in (3.6). 
We note that the expression (3.16) for $n=1$ coincides with the first two terms of the expansion in $\varepsilon$ of the exact result obtained in [31] (we explain below that the quantity $\Delta_{1}^{\prime}$ corresponds to $\delta-\gamma$ in the notation of [31]). For the case of the SNS model, critical dimensions of irreducible tensor operators are studied, for example, in [16].

Since the critical dimensions of the operators $\theta^{n}$ and $(\partial \theta)^{n}$ have been found, we can use the general expression (2.21) in the case of the structure functions (1.11) and pair correlators $(1.13)$.

The structure function $S_{2 n}(r)=\left\langle\left[\theta(x)-\theta\left(x^{\prime}\right)\right]^{2 n}\right\rangle$ is represented as a sum of pair correlators $\left\langle\theta(x)^{k} \theta\left(x^{\prime}\right)^{m}\right\rangle$ with fixed value of $k+m=2 n$ and equal canonical and critical dimensions $d_{F}^{\omega}=2 n d_{\theta}^{\omega}=-n, \Delta_{F}=2 n \Delta_{\theta}=n(-2+\varepsilon)$. Then from Eq. (2.21) with $m=0$ it follows:

$$
S_{2 n}(r) \simeq D_{0}^{-n} r^{n(2-\varepsilon)} f_{n}(M r)
$$

with certain, as yet unknown, scaling functions $f_{n}(M r)$.

The $n$th power of the dissipation rate is represented as a finite linear combination of basis operators (3.2) with definite critical dimensions $\Delta_{k}=k \varepsilon+\gamma_{k}^{*}$ given in (3.10), with the necessary condition $k \leq n$. Therefore, for the pair correlators (1.13) of the powers of the dissipation rate we obtain from (2.21):

$$
\left\langle E^{n}(x) E^{m}\left(x^{\prime}\right)\right\rangle=\nu_{0}^{n+m}\left\langle F_{n}(x) F_{m}\left(x^{\prime}\right)\right\rangle \simeq \sum_{k=0}^{n} \sum_{l=0}^{m}(\Lambda r)^{-\Delta_{k}-\Delta_{l}} f_{k, l}(M r),
$$

with certain scaling functions $f_{k, l}(M r)$ and the UV scale $\Lambda$ defined in (1.10). The leading term of the asymptotic behaviour of the expression (3.19) in the IR region $\Lambda r>>1$ is given by the contribution with minimal $\Delta_{k}+\Delta_{l}$, i.e.,

$$
\left\langle E^{n}(x) E^{m}\left(x^{\prime}\right)\right\rangle \simeq(\Lambda r)^{-\Delta_{n}-\Delta_{m}} f_{n, m}(M r) .
$$

We note that the structure functions (3.18) and the correlator (3.20) for $n=m=1$ are independent of the diffusivity coefficient, or, equivalently, of the UV scale $\Lambda$ (we recall that $\Delta_{1}=0$ ). This statement is an analog of the Second Kolmogorov hypothesis (independence of the viscosity coefficient in the inertial and energy-containing ranges) for the real fully developed turbulence, see e.g. [1] and [2]. Within the RG approach to the SNS model, the Second Kolmogorov hypothesis was established in [13], see also [12,14.

Expressions like (3.18), (3.20) can easily be written down for any single-time pair correlator, provided its canonical and critical dimensions are known. Let us give two more examples. For the second-rank irreducible tensors $E_{n}^{\prime}=\nu_{0} F_{n}^{\prime}$ with the operators $F_{n}^{\prime}$ given in (3.15) we obtain (dropping the vector indices):

$$
\left\langle E_{n}^{\prime}(x) E_{m}^{\prime}\left(x^{\prime}\right)\right\rangle=\nu_{0}^{n+m}\left\langle F_{n}^{\prime}(x) F_{m}^{\prime}\left(x^{\prime}\right)\right\rangle \simeq(\Lambda r)^{-\Delta_{n}^{\prime}-\Delta_{m}^{\prime}} f_{n, m}^{\prime \prime}(M r),
$$

and for the mixed correlator of the scalar $E^{n}$ and the tensor $E_{n}^{\prime}$ we have: 


$$
\left\langle E^{n}(x) E_{m}^{\prime}\left(x^{\prime}\right)\right\rangle=\nu_{0}^{n+m}\left\langle F_{n}(x) F_{m}^{\prime}\left(x^{\prime}\right)\right\rangle \simeq(\Lambda r)^{-\Delta_{n}-\Delta_{m}^{\prime}} f_{n, m}^{\prime}(M r),
$$

with the dimensions $\Delta_{n}, \Delta_{n}^{\prime}$ given in (3.10), (3.16) and certain scaling functions $f_{n, m}^{\prime}(M r)$, $f_{n, m}^{\prime \prime}(M r)$ (the prime is not a derivative here).

\section{OPERATOR PRODUCT EXPANSION AND ANOMALOUS SCALING}

From the viewpoint of the renormalization group, the expressions (2.21) and (3.18)-(3.22) for any functions $f(M r)$ correspond to IR scaling in the region $L \equiv M^{-1}, r>>l \equiv \Lambda^{-1}$ for arbitrary fixed value of $M r$, with definite critical dimensions $\Delta_{F}$. The inertial range $l<<r<<$ corresponds to the additional condition $M r<<1$, and representations like (1.11) and (1.13) should be understood as certain additional statements about the explicit form of the leading terms of the asymptotic behaviour for $M r \rightarrow 0$.

In the theory of critical phenomena, the asymptotic form of scaling functions for $M \rightarrow 0$ is studied using the well known Wilson operator product expansion (OPE), see e.g. [9.10]; the analog of $L \equiv M^{-1}$ is there the correlation length $r_{c}$. This technique is also applied to the theory of turbulence, see e.g. [12,13].

According to the OPE, the single-time product $F_{1}\left(x_{1}\right) F_{2}\left(x_{2}\right)$ of two renormalized operators at $\mathbf{x} \equiv\left(\mathbf{x}_{1}+\mathbf{x}_{2}\right) / 2=$ const, and $\mathbf{r} \equiv \mathbf{x}_{1}-\mathbf{x}_{2} \rightarrow 0$ has the representation

$$
F_{1}\left(x_{1}\right) F_{2}\left(x_{2}\right)=\sum_{\alpha} C_{\alpha}(\mathbf{r}) \bar{F}_{\alpha}^{R}(\mathbf{x}, \mathbf{t}),
$$

in which the functions $C_{\alpha}$ are the Wilson coefficients regular in $M^{2}$ and $\bar{F}_{\alpha}^{R}$ are all possible renormalized local composite operators of the type (3.2) allowed by symmetry, with definite critical dimensions $\Delta_{\alpha}$.

The renormalized correlator $\left\langle F_{1}\left(x_{1}\right) F_{2}\left(x_{2}\right)\right\rangle$ is obtained by averaging (4.1) with the weight $\exp S_{R}$, the quantities $\left\langle\bar{F}_{\alpha}^{R}\right\rangle=M^{d_{\alpha}} \nu^{d_{\alpha}^{\omega}} a_{\alpha}(g, M / \mu)$ involving dimensionless functions $a_{\alpha}(g, M / \mu)$ appear on the right hand side. Their asymptotic behaviour for $M / \mu \rightarrow 0$ is found from the corresponding RG equations and has the form

$$
\left\langle\bar{F}_{\alpha}^{R}\right\rangle \propto M^{\Delta_{\alpha}} .
$$

From the operator product expansion (4.1) we therefore find the following expression for the scaling function $f(M r)$ in the representation (2.21) for the correlator $\left\langle F_{1}\left(x_{1}\right) F_{2}\left(x_{2}\right)\right\rangle$ :

$$
f(u)=\sum_{\alpha} A_{\alpha}(u) u^{\Delta_{\alpha}}, \quad u \equiv M r,
$$

with coefficients $A_{\alpha}$, which are regular in $u^{2}$, generated by the Wilson coefficients $C_{\alpha}$ in (4.1), which are regular in $M^{2}$. 
The leading contributions for $u \rightarrow 0$ are those with the smallest dimension $\Delta_{\alpha}$ and in the $\varepsilon$ expansions they are those with the smallest $d_{\alpha} \equiv d\left[F_{\alpha}\right]$ for $\varepsilon=0$. We shall term the operators with $\Delta_{\alpha}<0$, if they exist, dangerous [12,13], as they correspond to contributions to (4.3) which diverge for $u \rightarrow 0$.

In the standard model $\phi^{4}$ of the theory of critical behaviour [10], there are no problem of dangerous operators within the $\varepsilon$ expansions, because in that model $\Delta_{\alpha}=n_{\alpha}+O(\varepsilon)$, where $n_{\alpha} \geq 0$ is the total number of fields and derivatives in $F_{\alpha}^{R}$. The operator $F=1$ has the smallest value $n_{\alpha}=0$, but it gives a contribution to (4.3) which is regular in $u^{2}$ and has a finite limit as $u \rightarrow 0$. The first nontrivial contribution is generated by the operator $\phi^{2}$ with $n_{\alpha}=2$, it has the form $u^{2+O(\varepsilon)}$ and only determines correction to the leading term generated by the operator $F=1$, which vanishes at $u \rightarrow 0$.

We note that for a Galilean invariant product $F_{1}\left(x_{1}\right) F_{2}\left(x_{2}\right)$, the right hand side of Eq.(4.1) can involve any Galilean invariant operator, including tensor operators, whose indices would be contracted with the analogous indices of the coefficients $C_{\alpha}$. Without loss of generality, it can be assumed that the expansion is made in irreducible tensors (see Sec.III for examples), so that only scalars contribute to the correlator $\left\langle F_{1} F_{2}\right\rangle$ because the averages $\left\langle\bar{F}_{\alpha}^{R}\right\rangle$ for nonscalar irreducible tensors are zero. For the same reason, the contributions to the correlator from all operators of the form $\partial F$ with external derivatives vanish owing to translational invariance.

In our case, contributions from the operators like $\theta^{n}$ with negative $d_{F}$ are also forbidden by the invariance of the correlators (3.18)-(3.22) with respect to the shift $\theta(x) \rightarrow \theta(x)+$ const of the field $\theta$.

The leading terms of the asymptotic behaviour of the scaling functions in (3.18)-(3.22) for $M r \rightarrow 0$ are therefore determined by the scalar operators $F_{n}=\left[\partial_{i} \theta \partial_{i} \theta\right]^{n}$ with the minimal canonical dimension $d_{F}=0$, see Sec. III. From the analysis of the diagrams it follows that the number of the fields $\theta$ in the operator $F_{n}$ entering into the right hand sides of the expansions (4.1) can never exceed the total number of the fields $\theta$ in their left hand sides. Therefore, only finite number of operators $F_{n}$ contributes to each operator product expansion in the model (2.1), and the asymptotic form of the scaling functions in (3.18)-(3.22) for $u \rightarrow 0$ is given by the expression:

$$
f(u)=\sum_{n=0}^{N} C_{n} u^{\Delta_{n}}+\ldots,
$$

where $N$ is the total number of the fields $\theta$ in the left hand sides, $C_{n}=C_{n}(\varepsilon, d)$ are numerical coefficients, and the dots stand for corrections of order $u^{2+O(\varepsilon)}$ vanishing as $u \rightarrow 0$. The leading term for $u \rightarrow 0$ is determined by the operator with minimal $\Delta_{n}$, i.e., with maximal $n$ (owing to the fact that the dimension $\Delta_{n}$ within the $\varepsilon$ expansion decreases monotonically with $n$, see $(3.10)$ ). As a result, for the scaling function $f_{n}$ in the representation (3.18) for 
the structure function $S_{2 n}$ we obtain:

$$
f_{n} \simeq \text { const } u^{\Delta_{n}} \quad \text { for } u \rightarrow 0,
$$

and for the correlators $(3.20)-(3.22)$ we have:

$$
f_{n, m}, f_{n, m}^{\prime}, f_{n, m}^{\prime \prime} \simeq \text { const } u^{\Delta_{n+m}} \quad \text { for } u \rightarrow 0
$$

with the critical dimensions $\Delta_{n}$ given in (3.10).

Therefore, we have derived expressions (1.11), (1.13), and relate the corresponding anomalous exponents to the critical dimensions of the composite operators $E^{n}(x)$.

\section{DISCUSSION AND CONCLUSION}

We have shown that the renormalization group combined with the operator product expansion establishes the existence of anomalous scaling in the model (1.7)-(1.9) for the advection of a passive scalar by a Gaussian velocity field and allows the corresponding anomalous exponents to be calculated in the form of series in $\varepsilon$.

The distinguishing feature of the model (1.7)-(1.9) that explains the origin of the anomalous scaling is the existence of dangerous composite operators with negative critical dimensions. They dominate the asymptotic behaviour of the scaling functions and lead to singular dependence of the correlation functions on the IR scale $M$ for $M \rightarrow 0$, in contrast to the standard models of critical phenomena, in which all the nontrivial operators have positive critical dimensions and only determine vanishing corrections to the leading finite contribution from the simplest operator $F=1$, see e. g. [10]. In contradistinction with the SNS model, the dangerous operators in the model (1.7)-(1.9) occur already for asymptotically small values of $\varepsilon$ and only finite number of these operators contribute to operator product expansion for any given correlation function.

The set of expressions (3.18)-(3.22), (4.4)-(4.6) gives the complete description of the IR asymptotic behaviour of the Green functions in the model (1.7)-(1.9): Eqs.(3.18)-(3.22) describe the asymptotic form of the structure functions and pair correlators in the IR region $L, r>>l \equiv \Lambda$ and determine their dependence on the UV scale $\Lambda$, while Eqs.(4.4)-(4.6) give the asymptotic form of the corresponding scaling functions upon additional restriction $M r<<1$ and determine the dependence on the IR scale $L \equiv M^{-1}$. All the critical dimensions $\Delta_{n}, \Delta_{n}^{\prime}$ entering into (3.18)-(3.22), (4.4)-(4.6) have been calculated in the second order of the $\varepsilon$ expansion, see (3.10), (3.16), while the critical dimensions of the model parameters, primary fields, and their powers have been found exactly, see (2.18), (2.19), (2.20), and (3.4). 
It should be stressed that the asymptotic expressions (4.5), (4.6) result from the fact that the critical dimensions $\Delta_{n}$ are negative and that $\left|\Delta_{n}\right|$ increases monotonically with $n$. This is obviously so within the $\varepsilon$ expansion, in which the sign and the $n$ dependence of the dimensions are determined by the first-order terms, while the higher-order terms are treated as small corrections. However, for finite values of $\varepsilon$ the higher-order terms can, in principle, change these features of the dimensions. Indeed, the $n^{3}$ contribution in the second-order approximation for $\Delta_{n}$ is positive, see e.g. (1.14), (1.15), and so $\Delta_{n}$ becomes positive, provided $n$ is large enough. Of course, this conclusion is based on the second-order approximation of the $\varepsilon$ expansion and is therefore not reliable: higher-order terms of the $\varepsilon$ expansion contain additional powers of $n$, and the correct analysis of the large $n$ behaviour of the dimensions $\Delta_{n}$ requires resummation of the $\varepsilon$ series with the additional condition that $n \varepsilon \simeq 1$, but we know of no model in which such a resummation has been performed.

The comparison of the Eqs.(3.18)-(3.22), (4.5), (4.6) with the corresponding expressions in [31-34] shows that $\Delta_{n}=-\rho_{2 n}$ in the notation of [33], $\Delta_{2}=-\Delta, \Delta_{1}^{\prime}=\gamma-\delta$ in the notation of [31], and $\Delta_{n}=-\Delta_{2 n}$ in the notation of [32]; our results for $\Delta_{n}, \Delta_{n}^{\prime}$ are in agreement with the results obtained in [31-34] for the structure functions $S_{2 n}(r)$ and the pair correlators (3.20), and in [31] for the correlators (3.21), (3.22) with $n=m=1$ within the first order of the expansions in $\varepsilon$ and $1 / d$.

It is noteworthy that the set of scalar operators $F_{n}=\left[\partial_{i} \theta \partial_{i} \theta\right]^{n}$ is "closed with respect to the fusion" in the sense that the leading term in the OPE for the pair correlator $\left\langle F_{n} F_{m}\right\rangle$ is given by the operator $F_{n+m}$ from the same family with the summed index $n+m$, see (3.20), (4.6). This fact along with the inequality $\Delta_{n}+\Delta_{m}>\Delta_{n+m}$, which is obvious from the explicit expressions for $\Delta_{n}$, can be interpreted as the statement that the correlations of the local dissipation rate in the model (1.7)-(1.9) exhibit multifractal behaviour, see 49 and [50]. We note that the same relation ensures the fulfillment of the Hölder inequality for the structure functions (1.11).

An important question is that of the universality of anomalous exponents, see e.g. [35]. It is clear from the RG analysis, that the exponents $\Delta_{n}$ do not depend on the choice of the correlator (1.9) (this correlator does not enter at all into the UV divergent diagrams which determine renormalization constants), and that they are insensitive to the specific form of the IR regularization in the correlator (1.8) (renormalization constants do not depend on the choice of the IR regularization, see e.g. [9]). However, the anomalous exponents can change if the function $\delta\left(t-t^{\prime}\right)$ in the correlator of the velocity field is replaced by some function with finite width, i.e., the velocity field has small but finite correlation time [36]. The RG approach to this problem will be presented elsewhere, and here we only mention another possible modification of the model (1.7)-(1.9), that of a "slow" velocity field. In this case, the function $\delta\left(t-t^{\prime}\right)$ in (1.9) is replaced by the unity, so that the velocity correlator is time independent. 
The RG analysis can be directly extended to this model to prove that its Green functions also exhibit anomalous scaling behaviour and the corresponding anomalous exponents can be calculated in the form of series in $\tilde{\varepsilon} \equiv \varepsilon+2$. The critical dimensions $\Delta_{\omega}=2-\tilde{\varepsilon} / 2$, $\Delta_{\theta}=-1+\tilde{\varepsilon} / 4$ are found exactly, and for the structure functions defined in (1.11) we have obtained:

$$
S_{2 n} \simeq D_{0}^{-n / 2} r^{n(2-\tilde{\varepsilon} / 2)}(M r)^{\tilde{\Delta}_{n}},
$$

where

$$
\tilde{\Delta}_{n}=-\tilde{\varepsilon} n(n-1) /(d+2)+O\left(\tilde{\varepsilon}^{2}\right) .
$$

The expressions (1.13) remain valid with the replacement $\Delta_{n} \rightarrow \tilde{\Delta}_{n}$. We note that the velocity field with the dimension $\Delta[\mathbf{v}]=1-\tilde{\varepsilon} / 2$ becomes dangerous for $\tilde{\varepsilon}>2$ (which corresponds to the IR divergence of the integral in (1.9) with $m=0$ ), and so become all its powers. However, these operators are not Galilean invariant and therefore give no contribution to the operator product expansions of the structure functions and correlators (1.13).

\section{ACKNOWLEDGMENTS}

The work was supported by the Russian Foundation for Fundamental Research (grant N 96-02-17-033) and by the Grant Center for Natural Sciences of the Russian State Committee for Higher Education (grant N 97-0-14.1-30). 


\section{REFERENCES}

[1] A. S. Monin and A. M. Yaglom, Statistical Fluid Mechanics (MIT Press, Cambridge, Mass., 1975), Vol.2.

[2] S. A. Orszag, Lectures on the Statistical Theory of Turbulence, in Fluid Dynamics (Les Houches Lectures) edited by R. Balian and J. L. Peube (Gordon and Breach, London,1973), p.235.

[3] R. A. Antonia, B. R. Satyaprakash, and A. K. M. F. Hussian, J.Fluid.Mech. 119, 55 (1982).

[4] F. Anselmet, Y. Gagne, E. Hopfinger, and R. A. Antonia, J.Fluid.Mech. 140, 63 (1984).

[5] V. R. Kuznetsov and V. A. Sabel'nikov Turbulence and Combustion (Hemisphere Publishing, New York, 1990).

[6] C. Meneveau and K. R. Sreenivasan, Phys. Rev. A 41, 2246 (1990).

[7] M. S. Borgas, Phys.Fluids A 4(9), 2055 (1992).

[8] N. N. Bogoliubov and D. V. Shirkov, Introduction to the Theory of Quantized Fields (Wiley, New York, 1980).

[9] J. Collins, Renormalization (Cambridge University Press, 1984).

[10] J. Zinn-Justin, Quantum Field Theory and Critical Phenomena (Clarendon, Oxford, 1989).

[11] C. De Dominicis and P. C. Martin, Phys. Rev. A 19, 419 (1979).

[12] L. Ts. Adzhemyan, N. V. Antonov, and A. N. Vasil'ev, Physics-Uspekhi 39 (12), 1193 (1996).

[13] L. Ts. Adzhemyan, N. V. Antonov, and A. N. Vasil'ev, Sov. Phys. JETP 68 (4), 733 (1989).

[14] N. V. Antonov and A. N. Vasil'ev, Theor. Math. Phys. 110, 97 (1997).

[15] L. Ts. Adzhemyan, N. V. Antonov, and T. L. Kim, Theor. Math. Phys. 100, 1086 (1994).

[16] L. Ts. Adzhemyan, S. V. Borisenok, and V. I. Girina, Theor. Math. Phys. 105, 1556 (1995).

[17] N. V. Antonov, S. V. Borisenok, and V. I. Girina, Theor. Math. Phys. 106, 75 (1996). 
[18] G. L. Eyink, Phys.Fluids 6, 3063 (1994).

[19] N. V. Antonov, S. V. Borisenok, and V. I. Girina, Theor. Math. Phys. 107, 456 (1996).

[20] L. Ts. Adzhemyan, A. N. Vasil'ev, and Yu. M. Pis'mak, Theor. Math. Phys. 57, 1131 (1983).

[21] L. Ts. Adzhemyan, A. N. Vasil'ev, and M. Hnatich, Theor. Math. Phys. 74, 115 (1988).

[22] R. H. Kraichnan, Phys. Fluids 7, 1723 (1964); 8, 575 (1965).

[23] S. Chen and R. H. Kraichnan, Phys.Fluids A 1, 2019 (1989).

[24] S. L. Woodruff, Phys. Fluids 6, 3051 (1994).

[25] L. Ts. Adzhemyan and N. V. Antonov, Renormalization Group in the Theory of Turbulence: Exactly Soluble Heisenberg Model, St Petersburg University Preprint SPbU-IP-97 (St Petersburg, 1997); to be published in Theor. Math. Phys.

[26] A. M. Obukhov, Izv. Akad. Nauk. SSSR, Geogr. Geofiz. 13, 58 (1949).

[27] R. H. Kraichnan, Phys. Fluids 11, 945 (1968).

[28] R. H. Kraichnan, Phys. Rev. Lett. 72, 1016 (1994); 78, 4922 (1997).

[29] R. H. Kraichnan, V. Yakhot, and S. Chen, Phys. Rev. Lett. 75, 240 (1995).

[30] G. Eyink, Phys. Rev. E 54, 1497 (1996);

G. Eyink and J.Xin, Phys. Rev. Lett. 77, 2674 (1996).

[31] M. Chertkov, G. Falkovich, I. Kolokolov, and V. Lebedev, Phys. Rev. E 52, 4924 (1995).

[32] M. Chertkov and G. Falkovich, Phys. Rev. Lett 76, 2706 (1996).

[33] K. Gawędzki and A. Kupiainen, Phys. Rev. Lett. 75, 3834 (1995).

[34] D. Bernard, K. Gawędzki, and A. Kupiainen, Phys. Rev. E 54, 2564 (1996).

[35] B. I. Shraiman and E. D. Siggia, C. R. Acad. Sci. 321, 279 (1995).

[36] M. Chertkov, G. Falkovich, and V. Lebedev, Phys. Rev. Lett. 76, 3707 (1996).

[37] A. L. Fairhall, O. Gat, V. L'vov, and I. Procaccia, Phys. Rev. E 53 (1996), 3518.

[38] V. Yakhot, Phys. Rev. E 55, 329 (1997).

[39] A. Pumir, B. I. Shraiman, and E. D. Siggia, Phys. Rev. E 55, R1263 (1997).

[40] O. Gat, V. L'vov, E. Podivilov, and I. Procaccia, Phys. Rev. E 55, R3836 (1997). 
[41] L. Ts. Adzhemyan, A. N. Vasil'ev, and M. Hnatich, Theor. Math. Phys. 58, 47 (1984).

[42] P. C. Martin, E. D. Siggia, and H. A. Rose, Phys. Rev. A 8, 423 (1973).

[43] H. K. Janssen, Z. Phys. B 23, 377 (1976).

[44] R. Bausch, H. K. Janssen, and H. Wagner, Z. Phys. B 24, 113 (1976).

[45] C. De Dominicis, J. Phys. (Paris) C1, 247 (1976).

[46] A. P. Prudnikov, Y. A. Brychkov, and O. I. Marichev, Integrals and Series (Gordon and Breach, New York, 1986), Vols.1 and 2.

[47] A. N. Vasil'ev, Functional Methods in Quantum Field Theory and Statistics (Leningrad University Press, Leningrad, 1976) [In Russian; English translation: Gordon and Breach, 1998].

[48] V. Yakhot, Z.-S. She, and S.A. Orszag, Phys.Fluids A 1(2), 289 (1989).

[49] B. Duplantier, A. Ludwig, Phys. Rev. Lett. 66, 247 (1991).

[50] G. L. Eyink, Phys. Lett. A 172, 355 (1993). 


\section{TABLES}

TABLE I. Canonical dimensions of the fields and parameters in the model (2.1).

\begin{tabular}{cccccccc}
\hline \hline$F$ & $\theta$ & $\theta^{\prime}$ & $\mathbf{v}$ & $\nu, \nu_{0}$ & $m, M, \mu$ & $g_{0}$ & $g$ \\
\hline$d_{F}^{k}$ & 0 & $\mathrm{~d}$ & -1 & -2 & 1 & $\varepsilon$ & 0 \\
$d_{F}^{\omega}$ & $-1 / 2$ & $1 / 2$ & 1 & 1 & 0 & 0 & 0 \\
$d_{F}$ & -1 & $\mathrm{~d}+1$ & 1 & 0 & 1 & $\varepsilon$ & 0 \\
\hline \hline
\end{tabular}

